\title{
Intracerebral Schwannoma Mimicking Meningioma: Case Report
}

\author{
Meihua Li, Jinhong Mei, Yiyun Li, Xueqin Tao, Tao Hong
}

Can J Neurol Sci. 2013; 40: 881-884

Intracranial schwannomas account for $8 \%$ of all primary brain tumors, approximately $80-90 \%$ of them being seen in the cerebellopontine angle in relation to the vestibular nerve. Schwannomas arising within brain parenchyma, unrelated to the cranial nerves, are extremely rare. To our knowledge, only 73 cases of intracerebral schwannoma have been described in the
English-language literature so far, ${ }^{1-5}$ but only six cases of intracerebral schwannoma mimicking meningioma have been reported $^{1-3}$ and there have been few reports regarding neuroradiological findings. Disagreement remains concerning the histogenesis of these tumors.

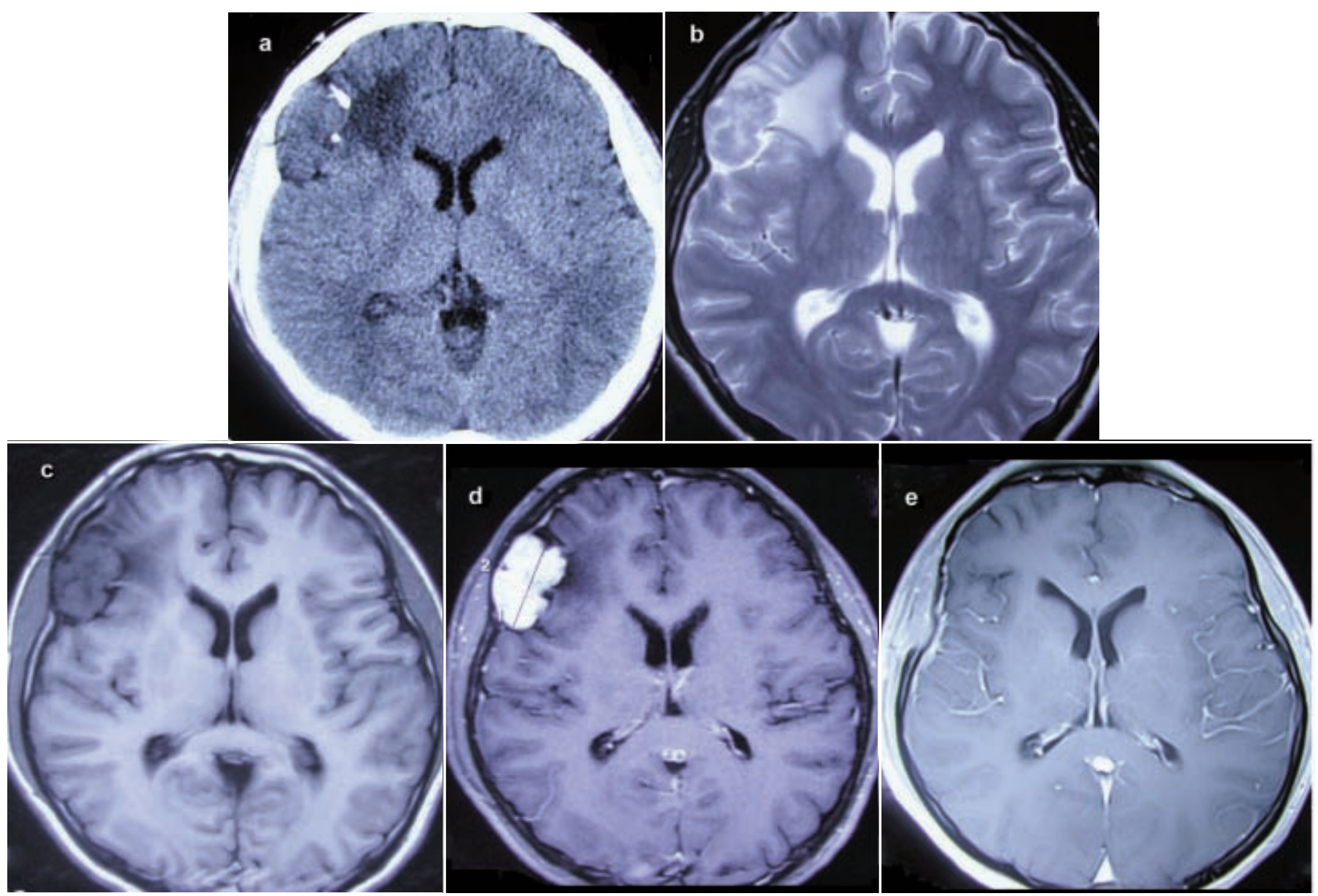

Figure 1: Computed tomograph (CT) and magnetic resonance imaging (MRI) of the tumor. a) CT scan shows a calcified, uniformly high-density lesion in the right frontal lobe, surrounded by brain edema. $b, c$ and d) MRI show a $3.5 \times 2.5 \mathrm{~cm}$, heterogeneously enhancing intra-axial lesion in the right frontal lobe with peritumoral edema and a dural-based attachment. e) follow-up MRI at 12 months showed no tumor recurrence.

From the Department of Neurosurgery (ML, YL, TH), Department of Pathology (JM, XT), the First Affiliated Hospital of Nanchang University, Nanchang, China. ReCEIVEd April 4, 2013. Final Revisions Submitted May 29, 2013.

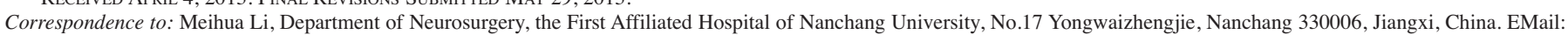
limeihua2000@sina.com. 
We herein describe a frontal intracerebral schwannoma with a dural-based attachment, occurring in a 19-year-old boy and, through a review of the literature, discuss the radiological features, histological and immunohistochemical findings, differential diagnoses and possible origin of this uncommon neoplasm.

\section{Case Report}

A 19-year-old boy was admitted to our department after suffering an epileptic seizure, five days before, that lasted for four minutes. He had never suffered any significant illness previously, no stigmata of neurofibromatosis was observed and there was also no family history of this disorder. Laboratory findings were normal. Neurological examination revealed no significant findings. Computerized tomography (CT) scans revealed a calcified, uniformly high-density lesion in the right frontal lobe, surrounded by brain edema (Figure 1a). Magnetic resonance images $(\mathrm{MRI})$ revealed a $3.5 \times 2.5 \mathrm{~cm}$, heterogeneously enhancing intra-axial lesion in the right frontal lobe with peritumoral edema and a dural-based attachment. (Figure 1b,1c and 1d). The preoperative diagnosis was meningioma. The patient underwent right frontal craniotomy with gross total resection of the tumor. Histopathological examination revealed schwannoma.

At surgery, no adherence to the dura was observed and a rim of intact pia was identified between the dura and tumor. The tumor-brain interface was indistinct in several regions; no clean arachnoid plane was identified. Postoperatively, the patient made a smooth recovery and no neurological deficits appeared. He has remained seizure-free since surgery. Follow-up MRI at 12 months showed no recurrence (Figure 1e).

\section{Pathological findings}

On pathological examination with hematoxylin and eosin stain, the tumor was composed of spindle-shaped cells with nuclear palisading (Antoni type A pattern; Figure 2a), loosely textured myxoid areas with decreased cellularity (Antoni type B pattern; Figure 2b) were also noted, and in some areas, both patterns mixed together (Figure 2c). Small aggregates of Schwann cells spread extensively into the surrounding brain tissue adjacent to the tumor nodule (Figure 2d), and significant calcification was seen in the surrounding brain tissue. Immunohistochemical staining was performed on formalin-fixed paraffin-embedded tissue. Primary antibodies against the following antigens were used: epithelial membrane antigen (EMA), S-100 protein, glial fibrillary acidic protein (GFAP), vimentin, CD56, CD34, calretinin (CR), neurofilament (NF), smooth muscle actin (SMA), desmin (Des) and Ki-67. The spindle cells was strongly positive for S-100 (Figure 3), CD56, and vimentin but negative for GFAP, EMA, CD34, CR, NF, SMA or Des. The Ki-67 labeling index was less than $1 \%$. A reticulin stain demonstrated pericellular reticulin deposition (Figure 4). Masson trichrome and Van Geison stains were also performed to demonstrate whether this tumor was myogenic or not. The results of both stains excluded the possibility.
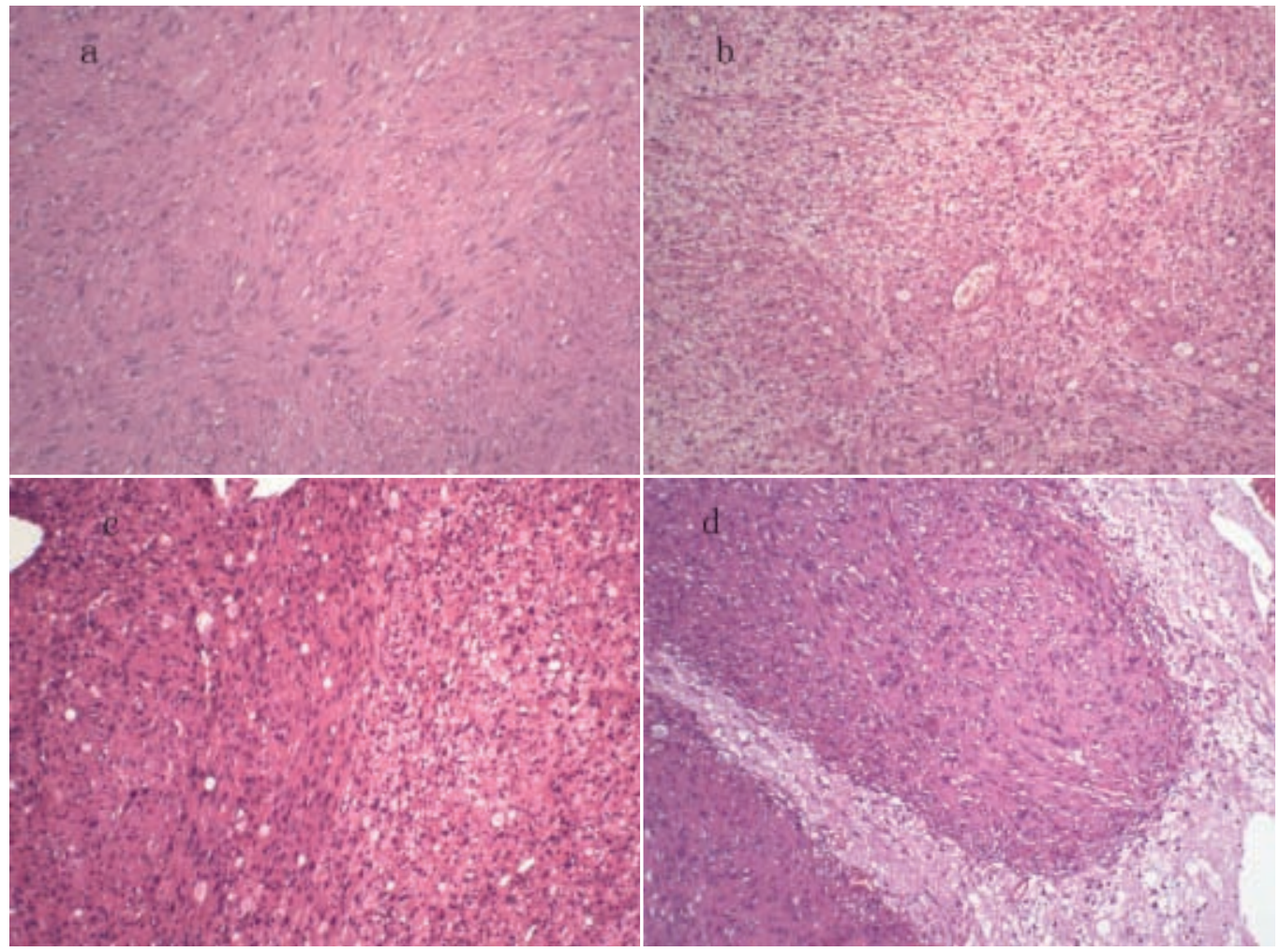

Figure 2: Histopathological micrographs (stain: hematoxylin and eosin, HEx100) show. a) interlacing bundles of spindle cells in a classic Antoni A pattern. b) a loosely textured myxoid appearance with decreased cellularity in a classic Antoni B pattern. c) a mixed area of both Antoni $A$ and $B$ types. $d)$ nodules of tumor cells extend into the surrounding parenchyma. 


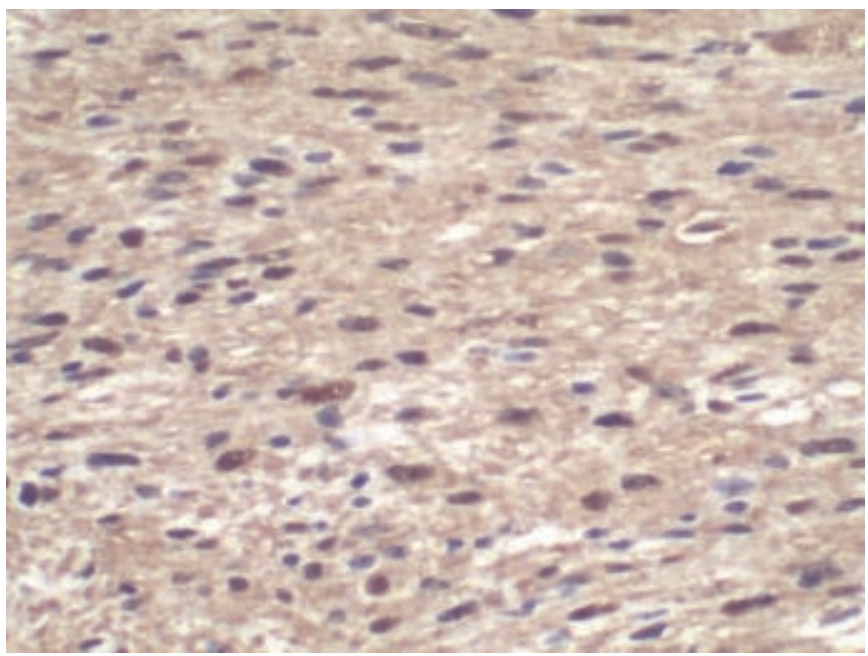

Figure 3: Immunohistochemical staining photomicrograph shows S-100 staining diffusely positive $(\times 400)$.

\section{Discussion}

Intracerebral schwannomas without any connection to the cranial nerves are rare. Seventy-four, including the present case, were reported in the English literature since 1966. ${ }^{1-5}$ There was no gender bias, age ranging from 4 to 78 -years-old, the peak incidence was in the second or third decade of life. Only seven cases occurred in patients with neurofibromatosis type 2. From the distribution within the brain parenchyma, supratentorial lesions are more common than infratentorial lesions.

Imaging characteristics of intracerebral schwannomas vary. Lesions of varying morphology, with non-homogenous contrast enhancement, associated with calcification, cyst formation or cystic degeneration, and peritumoral edema were reported to be common with intracerebral schwannomas. ${ }^{1-5}$ On CT, the tumors present as isodense or hypodense intra-parenchymal lesions in the frontal or temporal lobes, with a predilection for the periventricular white matter. They show uniform contrast enhancement and often have a cystic component. It was noted that the presence of a cyst together with the tumor appears to be a characteristic of intracerebral schwannoma, and cysts were documented histologically or by imaging studies in $34 \%$ of the 47 cases reviewed. ${ }^{1,2,4,5}$ Enhancement was described as homogeneous in $58 \%$ of 24 patients who underwent MRI with contrast. $^{1,2,5}$ Interestingly, $43 \%$ of 47 cases documented by imaging studies of intracerebral schwannomas showed peritumoral edema, represented by T2-weighted hyperintensity. ${ }^{1,3,5}$ The present case reported here presented on MRI as a superficially located, dural-attached tumor with contrast enhancement in the cerebrum, which mimicked a meningioma radiologically and clinically. Six similar cases have been reported, ${ }^{1,3,4}$ including three schwannomas in the temporal lobe, one frontoparietal schwannoma, one parasagittal schwannoma, and one schwannoma of the falx. It was interesting to note that dural tail sign could be identified in none of these cases, including our case.

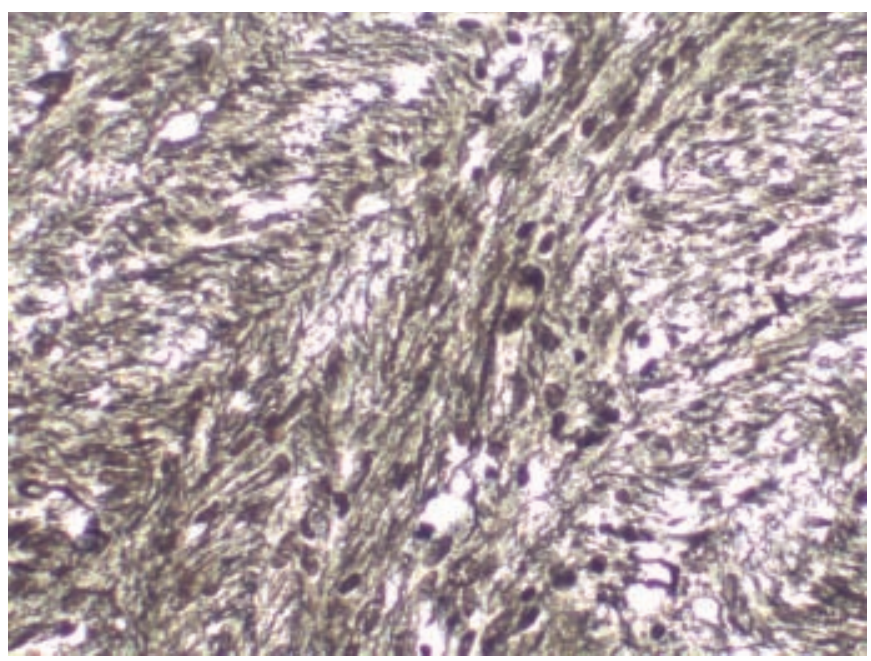

Figure 4: A reticulin stain demonstrated pericellular reticulin deposition $(\times 400)$.

In all but two cases, intracerebral schwannomas were described as "well-demarcated". An adult with a left frontal parasagittal schwannoma and an eight year-old boy with posterior right frontal schwannoma were reported to exhibit parenchymal invasion. ${ }^{4}$ To our knowledge, our case presented here is the third patient with invasive intracerebral schwannoma in the English literature so far. Because these tumors are so rare, the implications of this finding are unclear. However, the importance of obtaining regular follow-up should be recognized, because a recurrence is still possible although the margins appeared grossly clean at the end of the resection.

The preoperative diagnosis of an intracerebral schwannoma is difficult due to few characteristic radiological features and the low incidence. The differential diagnosis of an intracerebral schwannoma includes meningioma, piloctic astrocytoma, pleomorphic xanthoastrocytoma, ganglioglioma, and glioblastome multiforme. Microscopic differential diagnosis includes fibroblastic meningioma and solitary fibrous tumor from Antoni type A predominant schwannoma, and pilocytic/ pilomyxoid astrocytoma from Antoni type B predominant schwannoma. Sometimes it is very difficult to differentiate between these neoplasms based on the histological examination alone, as there is an overlap in their histological features. Therefore, either immunohistochemical or electron microscopic examination should be employed to make a correct diagnosis. ${ }^{3}$ No single immunoreactivity pattern is pathognomonic of schwannoma, and thus a combined use of immunostains (S100 protein, EMA, CD34, etc) is of great help in distinguishing schwannoma from its histological mimickers. ${ }^{1,3,5}$ Schwannomas are invariably $\mathrm{S}-100$ protein positive and meningiomas are EMA positive, whereas both antibodies are completely absent in solitary fibrous tumor. ${ }^{3,4}$ The present case and all the 24 previously reported cases of intracerebral schwannoma diagnosed with immunohistochemical stains had diffuse strong reactivity for S-100 protein., ${ }^{1,3,4}$ Fibroblastic meningioma have been reported to be often $\mathrm{S}-100$ protein positive, the rate being 
nearly $80 \% .^{1,3,4}$ Hence, S-100 protein alone might not reliably differentiate between fibroblastic meningioma and schwannomas. The present and all 15 previously reported cases in which EMA immunostains were performed for the diagnosis, revealed no reactivity for EMA., ${ }^{1,3}$ Several other immunostains (GFAP, CR, NF, SMA or Des) and special stainings (reticulin stain, Van Gieson and Masson) had been also performed for the differential diagnosis from other brain tumors of different sources in the present case.

The histogenesis of intracerebral schwannomas is unclear because Schwann cells are not normally found in the central nervous system except for the areas not associated with the cranial nerves. Several theories have been developed to explain their occurrence. First, Schwann cells of the perivascular nerve plexus in the subarachnoid space have been proposed to be the most likely source of intracerebral schwannoma. ${ }^{1-4}$ This hypothesis would explain the predilection of intracerebral schwannomas for superficial or deep periventricular sites. Second, mesenchymal multipotential cells are thought to be able to differentiate into Schwann cells according to developmental origin theory, which could account for their occurrence in children and young adults. However, to date, there is no conclusive evidence to support these hypotheses.

\section{Conclusion}

In conclusion, intracerebral schwannoma is a rare, benign neoplasm, characteristically occurring in young adults and children. The clinical, radiological, and histological differential diagnosis includes fibrous meningioma, astrocytoma, neurofibroma, and solitary fibrous tumor. Immunohistochemical staining is crucial for differential diagnosis. The implications of parenchymal invasion of intracerebral schwannoma are unclear. However, the importance of obtaining regular follow-up should be recognized.

\section{REFERENCES}

1. Paredes I, Jimenez Roldán L, Ramos A, Lobato RD, Ricoy JR. Intraparenchymal schwannomas: Report of two new cases studied with MRI and review of the literature. Clin Neurol Neurosurg. 2012;114(1):42-6.

2. Erongun U, Ozkal E, Acar O, Uygun A, Kocaogullar Y, Güngör S. Intracerebral schwannoma: Case report and review. Neurosurg Rev.1996;19:269-74.

3. Takei H, Schmiege L, Buckleair L, Goodman JC, Powell S. Intracerebral schwannoma clinically and radiologically mimicking meningioma. Pathol Int. 2005;55:514-9.

4. Bristol RE, Coons SW, Rekate HL, Spetzler RF. Invasive intracerebral schwannoma mimicking meningioma in a child. Childs Nerv Syst. 2006;22:1483-6.

5. Luo W, Ren X, Chen S, Liu H, Sui D, Lin S. Intracranial intraparenchymal and intraventricular schwannomas: Report of 18 cases. Clin Neurol Neurosurg. 2013 Jul;115(7):1052-7. 